\title{
Estudo de um aquecedor solar para um ciclo de geração de potência
}

\author{
Davi Pavis Parrol, Eduardo Lobo Lustosa Cabral'; Thadeu das Neves Conti ${ }^{l}$ \\ $\bowtie$ davi.parro@gmail.com
}

1. Instituto de Pesquisas Energéticas e Nucleares-IPEN.

\author{
Histórico do Artigo: \\ Recebido em: 26 de fevereiro de 2018 Aceito em: 16 de outubro de 2018 Publicado em: 28 de dezembro de 2018
}

Resumo: Conhecida a crescente demanda por energia, é de suma importância que sejam criados meios de otimizar sua geração e proporcionar mais economia. 0 presente trabalho trata de um ciclo de geração de potência por meio de um aquecedor solar. 0 objetivo é sintetizar, baseado na literatura, uma modelagem matemática que forneça parâmetros de funcionamento para serem confrontados com medidas experimentais. Palavras-chave: Termodinâmica, aquecedor, simulação, programa, ciclo, potência.

\section{Study of a solar heater for a power generation cycle}

Abstract: As the demand for energy is growing, it's very important that ways of optimizing and saving energy are created. This work is about a power generation cycle through a solar heater. The goal is synthesize, based on the literature, a mathematical modeling that provides working parameters that will be confronted with experimental measures.

Keywords: Thermodynamics, heater, simulation, software, cycle, power.

\section{Estudio de un calentador solar para un ciclo de generación de potencia}

Resumen: A medida que la demanda de energía crece, es muy importante que se creen formas de optimizar y ahorrar energía. Este trabajo es sobre un ciclo de generación de energía a través de un calentador solar. El objetivo es sintetizar, basado en la literatura, un modelado matemático que proporcione parámetros de funcionamiento para ser enfrentados con medidas experimentales.

Palabras clave: Termodinámica, calentador, simulación, software, ciclo, potencia. 
Projeto de um aquecedor solar para um ciclo de geração de potência

\section{INTRODUÇÃ0}

Este projeto tem por objetivo elaborar uma rotina computacional que descreva o funcionamento e os fenômenos de um aquecedor solar que, futuramente, será construído e testado nas dependências do Instituto de Pesquisas Energéticas e Nucleares (IPEN). Sua finalidade é a aquecer o fluido de trabalho (ar) de um ciclo termodinâmico de potência utilizando energia solar (Silva, 2014), que não emite gases poluentes e se mostra em abundância conforme a incidência de radiação solar na localidade referida (MARTINS, 2005) e (Ferrer et Ribeiro, 2011).

\section{MATERIAL E MÉTODOS}

0 principal objetivo da rotina computacional idealizada é que ela descreva os fenômenos envolvidos nos processos do ciclo termodinâmico a ser estudado, e retorne as condições de entrada e saída do fluido de cada processo (M0M0I, 2005). A análise das simulações e dos parâmetros de saída, desta forma, tornará viável a seleção de materiais para a futura construção e testes do ciclo.

Para tanto, será utilizado o programa computacional Scilab para a implementação da rotina. 0 Scilab é um software livre, de código aberto, voltado para análise numérica, que possui uma ampla de biblioteca de funções, e pode ser usado para muitas aplicações científicas.

Foram extraídos da literatura disponível modelos teóricos que descrevem os fenômenos de cada processo do ciclo e, assim, foi elaborada rotina que calcula as pressões e temperaturas de cada estado do ciclo (Huang, 1998). Assim, foram aplicados conhecimentos teóricos sobre Termodinâmica e utilizados numa aplicação de pesquisa e desenvolvimento, prestando-se serviço ao Centro de Engenharia Nuclear (CEN) do IPEN. (KALOGIROU, 2009)

0 esquema do ciclo é exposto a seguir (Figura 1). 


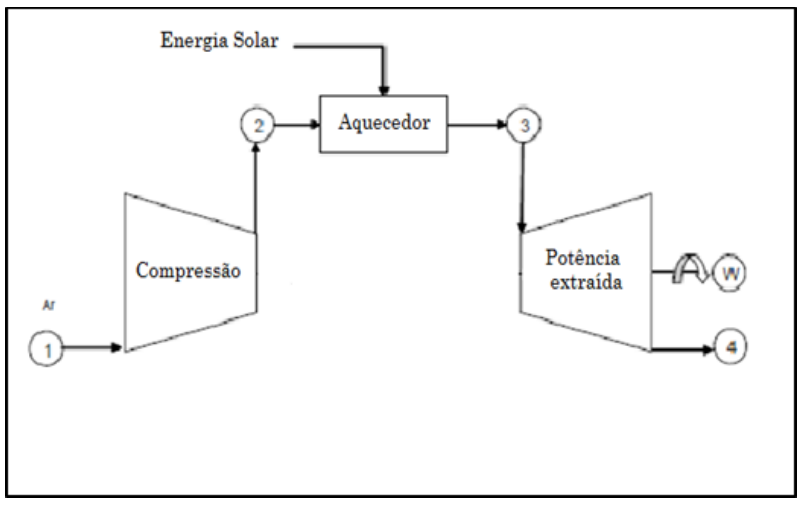

Figura 1. Esquema do ciclo de geração de Potência Fonte própria.

0s processos em cada equipamento são descritos a seguir:

- Processo 1-2, Compressão Isotérmica. A compressão é realizada de forma lenta 0 suficiente para que este processo possa ser aproximado pela hipótese de temperatura constante. A razão de compressão é pequena e pode ser feita à noite, pois não necessita de radiação solar.

- Processo 2-3, Aquecimento. Nesse processo o ar é aquecido usando a energia solar e atingindo altas temperaturas. 0 aquecedor solar, objeto deste trabalho, é o equipamento que realiza esse processo.

- Processo 3-4, Extração de Potência. Nesse processo converte-se a energia fornecida ao ar em energia mecânica, que pode vir a ser convertida em energia elétrica.

\section{AQUECEDOR SOLAR SEM PERDAS DE CALOR}

Inicialmente, foi feito o modelo do aquecedor solar (Processo 2-3) como um equipamento ideal, ou seja, sem perda de calor por conveç̧ão e radiação (DUFFIE, 1980). Para modelar o perfil de temperatura do ar, foram feitas as seguintes hipóteses simplificadoras:

- Sem perdas de calor;

- Sem perdas por atrito;

- 0 ar é tratado como gás perfeito;

- Regime permanente;

- 0 calor proveniente da radiação solar é uniforme por toda a superfície; 
- Vazão em volume constante;

Como parâmetros de entrada (que serão decididos na construção do ciclo) tem-se as propriedades do ar (calor específico, temperatura de entrada, pressão de entrada, coeficiente de difusividade térmica), o comprimento do duto pelo qual 0 ar será aquecido, vazão em volume, intensidade da radiação solar, dimensões da seção transversal do duto (a e b).

Da primeira lei da termodinâmica (P0TTER, et al., 2006) aplicada ao duto nesta primeira abordagem, obtém-se a seguinte relação:

$$
Q=m * c p *(T 3-T 2)
$$

Onde:

Q: taxa de calor proveniente da radiação solar $(\mathrm{kW})$;

m: vazão mássica de ar insuflado $(\mathrm{kg} / \mathrm{s})$;

$\mathrm{C}_{\mathrm{p}}$ : calor específico do ar ( $\left.\mathrm{kJ} / \mathrm{kgK}\right)$;

$\mathrm{T}_{2}$ : Temperatura de saída da compressão isotérmica(K);

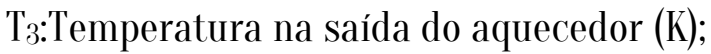

Fazendo uso do software Scilab, foi traçado o perfil da temperatura de saída do aquecedor, $T_{3}$, obtendo-se o perfil linear conforme esperado e exposto a seguir (Figura 2).

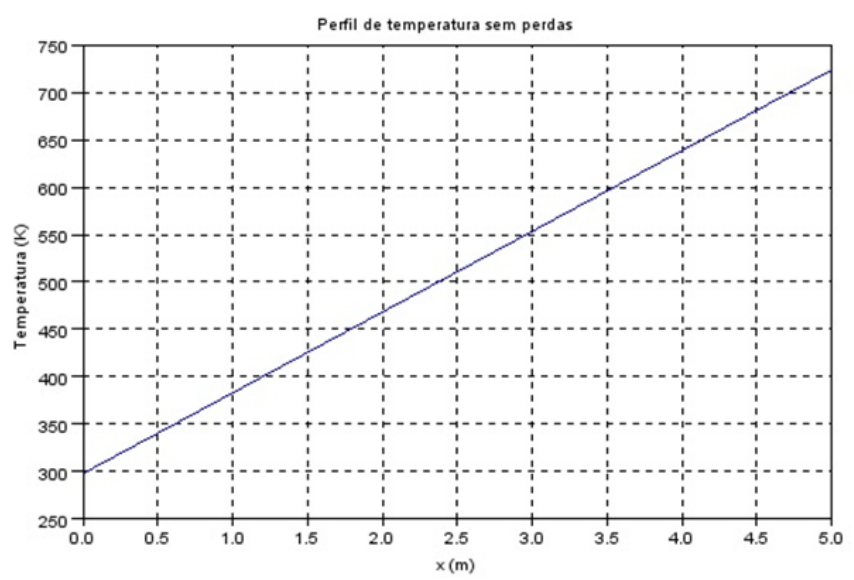

Figura 2. Temperatura ao longo do comprimento do duto sem perdas Fonte própria

0 eixo das abscissas representa o comprimento do duto escolhido aleatoriamente (nesta simulação, 5 metros). Este gráfico confirma o modelo teórico para as hipóteses assumidas num primeiro momento. Sobre a pressão, como 0 ar é admitido como gás perfeito, a pressão na saída do aquecedor, $P_{3}$, é modelada da seguinte forma: 
$P 3=P 2 *(T 3 / T 2)$

$\mathrm{P}_{3}$ : Pressão na saída do aquecedor (kPa);

$\mathrm{P}_{2}$ : Pressão na entrada do aquecedor $(\mathrm{kPa})$

Segue o perfil da pressão ao longo do duto (Figura 3).

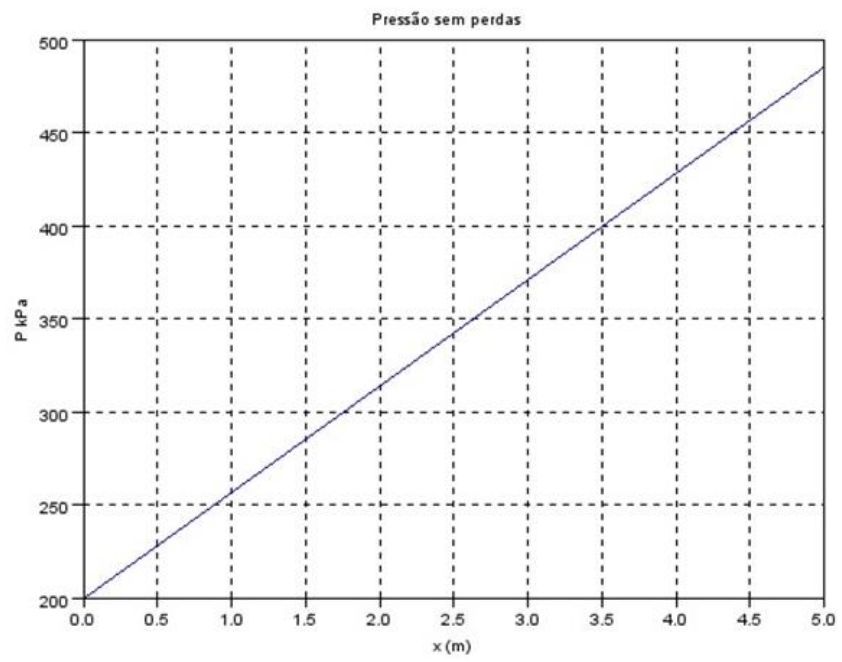

Figura 3. Pressão ao longo do comprimento do duto sem perdas Fonte própria

Pode-se observar que a pressão segue um perfil linear, conforme esperado do modelo. Vale lembrar que a primeira abordagem, sem perdas, feita neste capítulo foi apenas uma forma didática de se inserir no projeto, e que nos capítulos seguintes as perdas por convecção e radiação, muito relevantes no ciclo e até aqui desprezadas, serão consideradas.

\section{AQUECEDOR SOLAR COM PERDAS DO AQUECEDOR}

Assumidas as hipóteses do item anterior, para traçar-se o perfil da temperatura ao longo do duto, deve-se avaliar o tipo de escoamento de que se trata o problema. Além das hipóteses do item anterior, adiciona-se mais uma, de que a perda de carga do escoamento do ar é insignificante. 
Projeto de um aquecedor solar para um ciclo de geração de potência

Avaliando-se o número de Reynolds (Incropera, et al, 2003) deste escoamento (tendo como base os parâmetros de entrada citados), pela relação:

$$
\operatorname{Re}=(4 * m) /(\pi * d h * \mu)
$$

Onde:

m: vazão em massa $(\mathrm{kg} / \mathrm{s})$;

$\mathrm{d}_{\mathrm{h}}$ : diâmetro hidráulico (m);

$\mu$ : viscosidade dinâmica do ar $\left(\mathrm{Ns} / \mathrm{m}^{2}\right)$;

Vale lembrar que o diâmetro hidráulico é obtido da seguinte forma:

0 duto pelo qual o ar escoa é proposto conforme a Figura 4 a seguir.

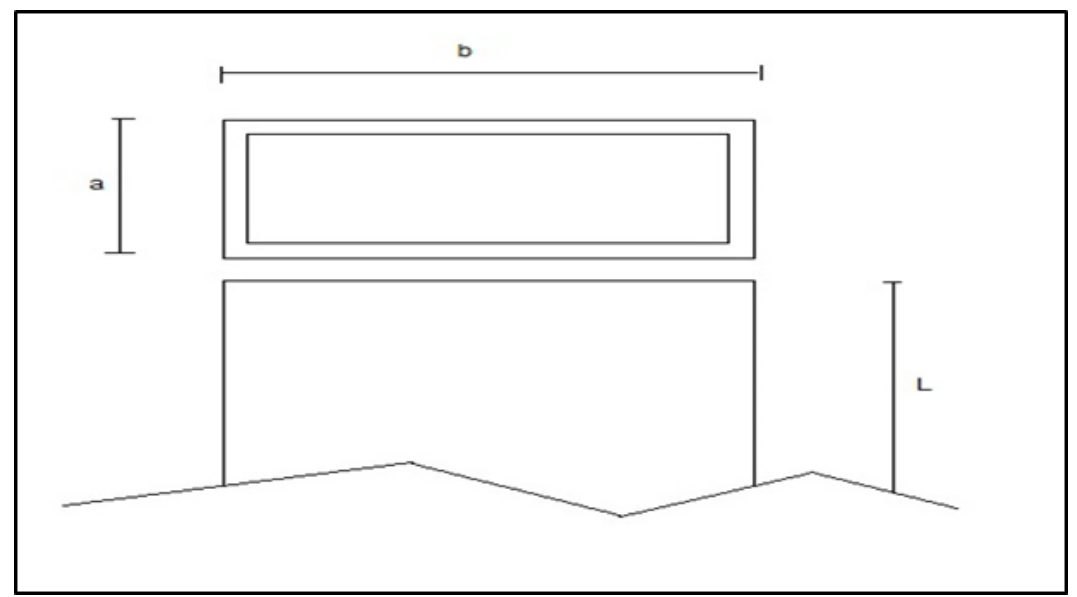

Figura 4. Vistas do duto (fora de escala)

Fonte própria

0 diâmetro hidráulico (MUNSON, 2002) é calculado por:

Diâmetro Hidráulico, $d h=4 * a * b /[2 *(a+b)]$

Pode-se observar, efetuando os cálculos, que o escoamento é turbulento, tendo seu Reynolds 12464,394. (Incropera, et al, 2003).

No caso deste projeto, $\mathrm{a}=10 \mathrm{~mm}$ e $\mathrm{b}=200 \mathrm{~mm}$.

0 balanço térmico deste escoamento é esquematizado na Figura 5 a seguir. 


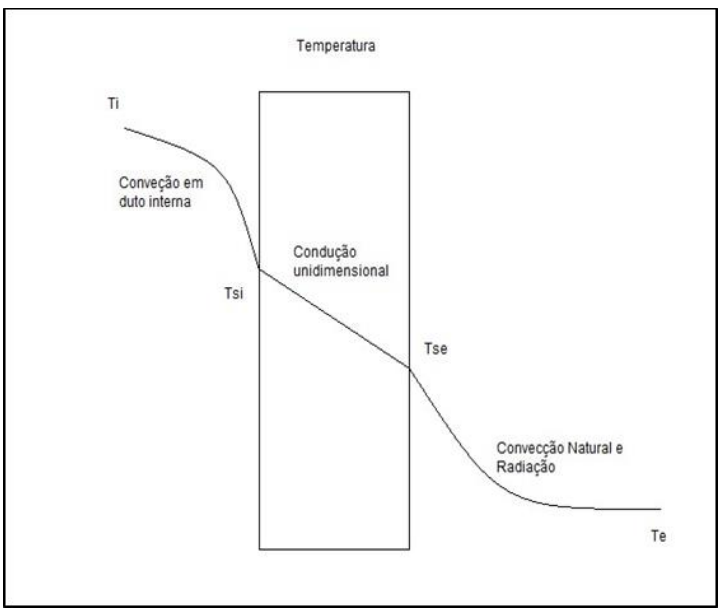

Figura 5. Esquema do balanço térmico

Fonte própria

A partir deste esquema de balanço térmico e da primeira lei da termodinâmica, obtém-se as equações que regem o escoamento. Estas equações são aplicadas a pequenas partições, dx, do comprimento, L, do duto, desta forma, calculando as temperaturas em cada partição do duto, o que traça o perfil de temperaturas.

Fazendo uso da hipótese de que a energia do sistema não varia e que o mesmo encontra-se em regime permanente, as relações aplicadas em cada pequena partição do duto são explicitadas a seguir (divisão em n volumes de controle conforme Figura 6 abaixo).

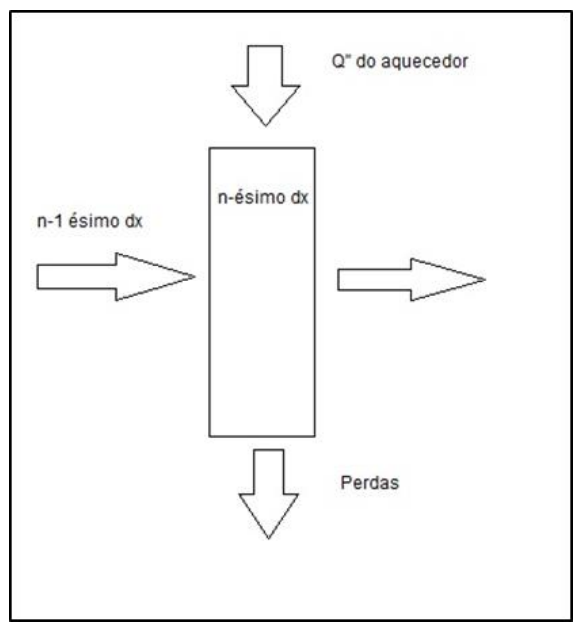

Figura 6. Partição em volumes de controle Fonte própria

- Perda por convecção interna = perda por condução 
Projeto de um aquecedor solar para um ciclo de geração de potência

Relação: $h i n *(T i-T s i)=k v i d r o *(T s i-T s e) / e$

Onde:

$\mathrm{h}_{\text {in: }}$ Coeficiente de troca de valor por convecção interna do duto $\left(\mathrm{W} / \mathrm{m}^{2} \mathrm{~K}\right)$;

$\mathrm{T}_{\mathrm{i}}$ : Temperatura do fluido na n-ésima partição do duto (K);

$\mathrm{T}_{\mathrm{si}}$ : Temperatura da parede interna do duto (K);

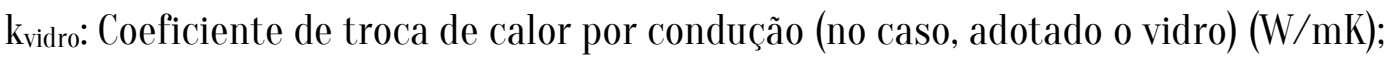

e: espessura do duto (no caso, assumida 10mm) (m);

$\mathrm{T}_{\mathrm{se}}$ : Temperatura da parede externa do duto $(\mathrm{K})$;

0 coeficiente $h_{\text {in }}$ é obtido da seguinte forma:

Tendo sido avaliado o escoamento como turbulento, obtém-se 0 adimensional Nusselt, Nu, por:

$$
N u=0,027 * R e 0,8 * \operatorname{Pr}(1 / 3)
$$

De posse do adimensional, Nu, Calcula-se $h_{\text {in }}$ por:

Relação: $\operatorname{hin}=N u * k a r / b$

- Perda por condução = perda por convecção natural + radiação

Relação:

$$
\begin{aligned}
& \left(\frac{k v i d r o}{e}\right) *(T s i-T s e)=\sigma * \varepsilon *(\text { Tse } 4-\text { Tvizinhança4 })+\text { he } *(\text { Tse }- \\
& T \infty)
\end{aligned}
$$

A espessura do vidro, e, é aqui assumida como 10mm.

- Calor recebido do aquecedor = perdas por convecção natural e radiação

Relação:

$$
\begin{aligned}
& m * c p *(T n-T n-1)=0,001 * 2 * n * d x * b *[Q "-\sigma * \varepsilon * \\
& (\text { Tse } 4-\text { Tvizinhança4 })+h e *(T s e-T \infty)]
\end{aligned}
$$

(A multiplicação por 2 no lado direito da igualdade se deve ao fato de as duas faces mais achatadas do duto perderem calor por convecção e radiação por terem maior área). 
- Cálculo do coeficiente de perda de calor por convecção natural, $\mathrm{h}_{\mathrm{e}}$.

Dadas as condições iniciais e de entrada deste projeto, o coeficiente he é determinado por etapas. Inicialmente determina-se o adimensional Rayleigh por:

$$
R a=g * \beta *(\text { Tse }-T \infty) /(\vartheta * \alpha)
$$

Onde:

g: gravidade $\left(\mathrm{m} / \mathrm{s}^{2}\right)$;

$\beta$ : coeficiente de expansão volumétrica $\left(\mathrm{K}^{-1}\right)$;

$\alpha$ : difusividade térmica $\left(\mathrm{m}^{2} / \mathrm{s}\right)$;

$\vartheta$ : viscosidade cinemática do ar $\left(\mathrm{m}^{2} / \mathrm{s}\right)$;

A partir do adimensional Ra, obtém-se o Nusselt, Nuex, pela relação:

Nuex $=\{0,825+[0,837 * \operatorname{Ra}(1 / 6)] /[1+(0,492 / \operatorname{Pr})(9 / 16)](8 / 27)\} 2$

Onde Pr é o adimensional de Prandtl.

Então se determina he, por

Relação: $h e=N u e x * k a r / L$

Pode-se observar que as equações de (5) a (12) formam um sistema não linear de equações algébricas (Fox, 2004). Sendo difícil e inviável a resolução analítica deste sistema, utiliza-se o software Scilab para a sua resolução.

Vale ressaltar que este sistema é resolvido para as n partições dx do comprimento do duto. A resolução deste sistema plota o seguinte comportamento para o perfil de temperatura ao longo do comprimento do duto (Figura 7). 


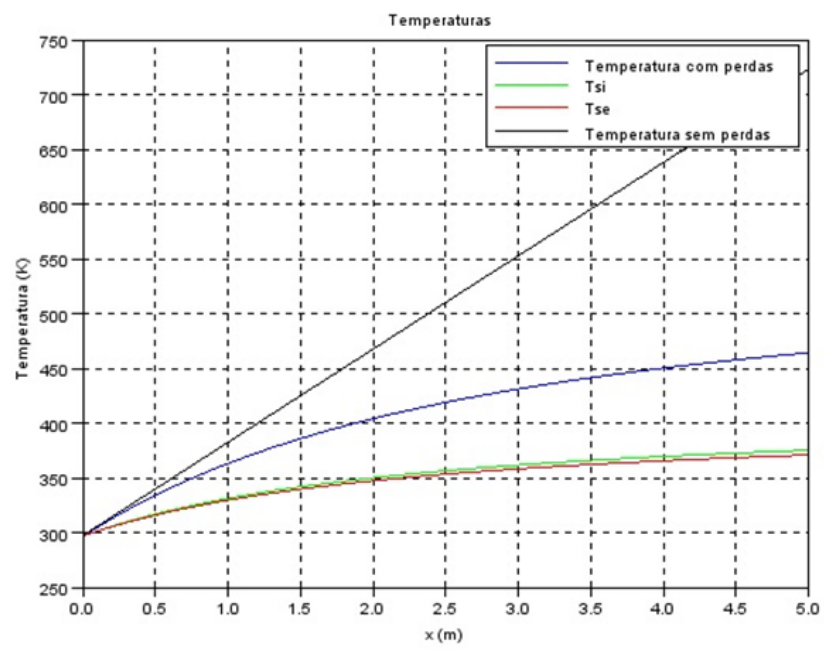

Figura 7. Perfil de temperaturas com as perdas do aquecedor Fonte própria

Vale ratificar que:

Linha azul: Temperatura do ar com perdas;

Linha preta: Temperatura do ar sem perdas;

Linha verde: Temperatura da parede interna do duto;

Linha vermelha: Temperatura da parede externa do duto;

Lembrado hipótese de que o ar comporta-se como gás ideal, pode-se traçar também a pressão ao longo do duto, que tem o formato de curva parecido com a da temperatura (Figura 8 a seguir).

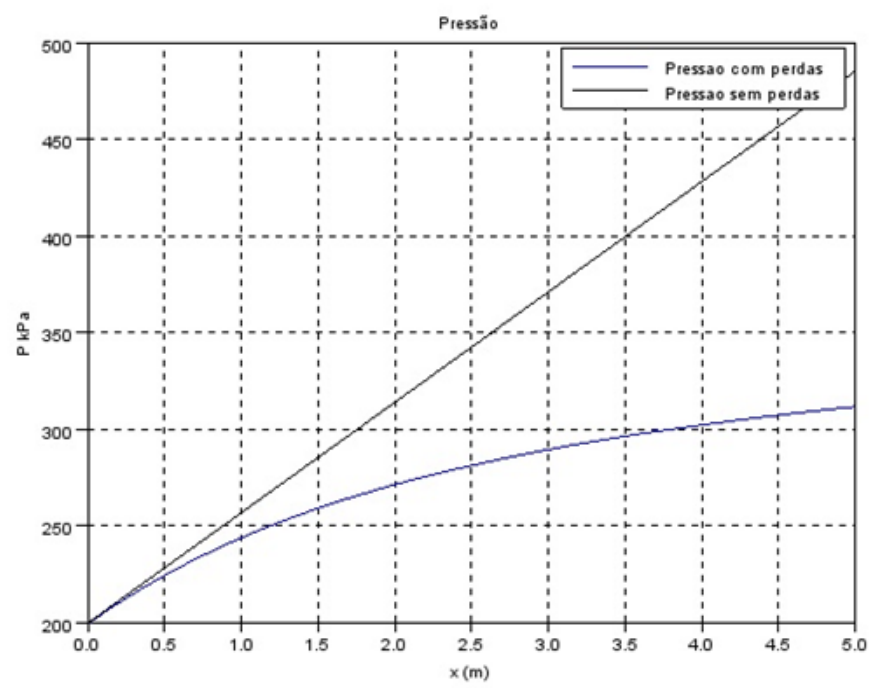

Figura 8. Pressão com perdas do aquecedor Fonte própria 
Linha azul: Pressão com perdas do aquecedor;

Linha Preta: Pressão sem perdas do aquecedor.

\section{CÁLCULO DO RENDIMENTO DO CICLO}

0 rendimento do ciclo de potência estudado é calculado a seguir:

Relação: $\eta$ ciclo $=($ Wcompressão + Wturbina $) /$ Qquecedor

Fazendo as manipulações algébricas convenientes,

Cada termo do cálculo do rendimento é explicitado a seguir:

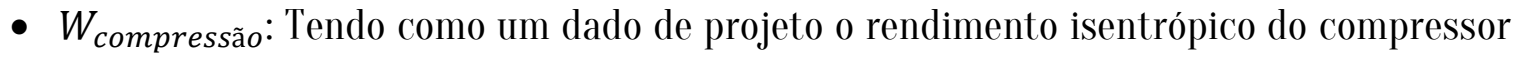
de 0.9 , a potência requerida na compressão isotérmica é dada por:

Wcompressão $=m * R *$ Tin $* \ln (P 1 / P 2) / \eta$ compressor

- $W_{\text {turbina }}$ : Também como dado de projeto, o rendimento isentrópico é assumido como 0.8, sua potência fornecida é assim calculada:

Relação: $\eta$ turbina $=(T 3-T 4) /(T 3-T 4 s)$

Onde

$$
T 4 s=T 3 *(P 4 / P 3)^{\wedge}[(k-1) / k]
$$

A partir daí, infere-se que:

$$
\text { Wturbina }=m * c p *(T 3-T 4)
$$

- 0 insumo energético fornecido do aquecedor é dado pela equação (1). (SONNTAG, et al., 1995). 
Projeto de um aquecedor solar para um ciclo de geração de potência

\section{RESULTADOS E DISCUSSÃ0}

A rotina computacional escrita no software Scilab fornece resultados coerentes e que evidenciam que as hipóteses simplificadoras assumidas são razoáveis e não produzem discrepâncias do modelo físico.

Para exemplificar isso, é exibido abaixo o perfil de temperatura das temperaturas das paredes interna e externa do duto. Pode-se inferir que há uma pequena diferença entre elas (com a temperatura da parede interna sempre maior que a da parede externa), resultante da perda de calor por condução através da espessura do material do duto. Entretanto, como a espessura é pequena, sua resistência térmica também, resultando numa diferença pequena de temperaturas mostrada em detalhe (Figura 9).

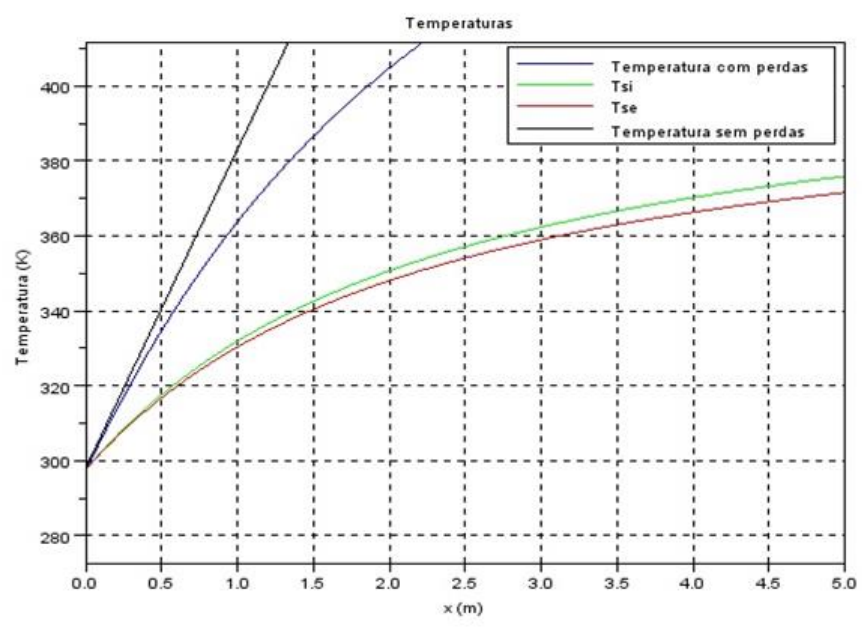

Figura 9. Diferença entre Tsi e Tse em detalhe Fonte própria

Os indicadores do ciclo de geração de potência variam de acordo com os seus dados de entrada. Para avaliar o comportamento deste ciclo, foi estipulado que as temperaturas de referência assumidas para o cálculo das trocas de calor por convecção e radiação, $T_{\infty}$ e $T_{\text {vizinhança }}$ respectivamente eram iguais a $298.15 \mathrm{~K}$, bem como a temperatura do ar na entrada do compressor, $T_{1}$. Sua pressão $P_{1}$ é de $100 \mathrm{kPa}$. Admite-se também que a razão de compressão do compressor isotérmico é 2 .

Para o projeto do aquecedor solar, deve-se previamente estipular a potência que se requer extrair deste ciclo termodinâmico. Nesse projeto, uma das variáveis mais importantes é o comprimento do duto, L. Para efeito didático, foi considerado um comprimento de $5 \mathrm{~m}$ nos cálculos até aqui realizados, entretanto esse comprimento deve ser dimensionado para a 
finalidade de extração de energia solar, bem como o concentrador solar que amplificará a intensidade de radiação solar imprimida no aquecedor (aqui admitida como $1 \mathrm{~kW} / \mathrm{m}^{2}$ ).

Mantendo-se a intensidade de radiação constante a $1 \mathrm{~kW} / \mathrm{m}^{2}$, e variando-se 0 comprimento do duto, pode-se observar que 0 rendimento deste ciclo apresenta 0 comportamento assintótico (obtido por regressão), conforme exposto na Figura 10 a seguir.

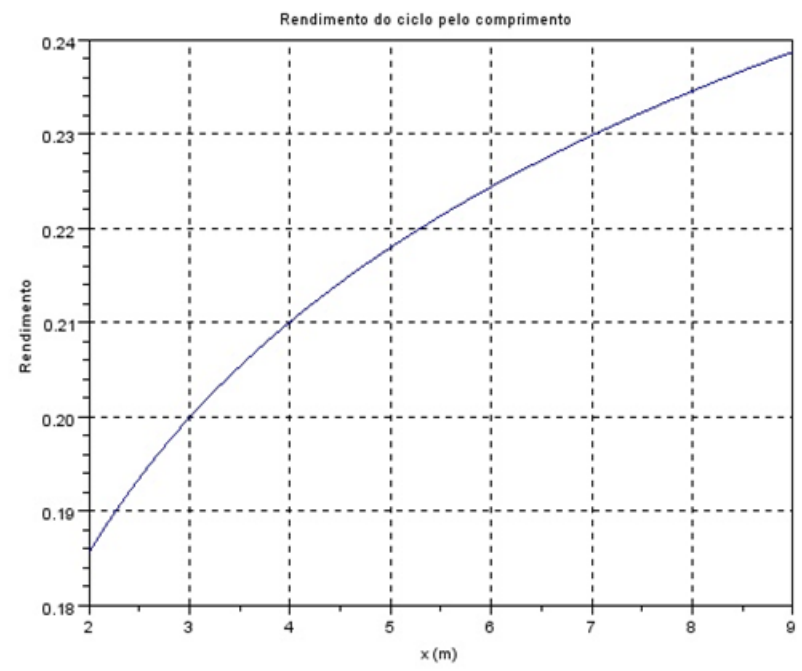

Figura 10. Rendimento do ciclo pelo comprimento do duto Fonte própria

No caso do comprimento de $5 \mathrm{~m}$ e intensidade de $1 \mathrm{~kW} / \mathrm{m}^{2}$, a potência requerida na compressão é de $0.31 \mathrm{~kW}$, a potência gerada na turbina é de $0.49 \mathrm{~kW}$ e 0 calor transferido do aquecedor é de $0.78 \mathrm{~kW}$.

Para os próximos passos desse projeto, será também requerido otimizar o material do duto do aquecedor, visando reduzir as perdas por convecção e radiação, bem como por condução em sua parede, o que renderá novos dados de entrada do projeto para os quais a rotina computacional elaborada atende e retorna o comportamento do ar no ciclo conforme esperado.

\section{CONCLUSÃ0}


Projeto de um aquecedor solar para um ciclo de geração de potência

Do exposto até aqui, pode-se concluir que, conforme descrito na literatura, a simulação do modelo utilizado neste trabalho fornece resultados que são passíveis de serem utilizados para comparação de dados teóricos e parâmetros de funcionamento do aquecedor.

Este trabalho pode servir de subsídio para a futura implementação prática do aquecedor, e servirá como balizador, ou seja, como calibrador. (GIL, 2010).

\section{REFERÊNCIAS BIBLIOGRÁFICAS}

DUFFIE, J.; BECKMAN, W.. Solar Engineering of Thermal Processes. $2^{\mathrm{a}}$ ed. Madison, WI: John Wiley \& Sons, Inc.1980 FERRER SILVA, I. A.; RIBEIR0, A. R.. Gestão Ambiental e Sustentabilidade. 1ª ed. Cuiabá: EduFMT, 2011.

FOX, R.W.; McDonald, A.T.; Pritchard, P.J.. Introdução à Mecânica dos Fluidos. 6a ed. Rio de Janeiro: LTC, 2004.

GIL, A. C.. Como elaborar projetos de pesquisa. $4^{\mathrm{a}}$ ed. São Paulo: Atlas, 2010.

Huang, F. F.. Engineering Thermodynamics: Fundamentals and Applications. 2a ed. New York: Maximillan, 1998.

INCROPERA, F. P.; DEWITT, D. P.. Fundamentos de Transferência de Calor e Massa. 5a Ed. Rio de Janeiro: LTC, 2003.

KALOGIROU, S. A.. Solar energy engineering: process and systems. 1 $^{\mathrm{a}}$ ed. San Diego (EUA): Elsevier, 2009.

MARTINS, F. R. et al.. Mapas de Irradiação Solar para o Brasil - Resultados do Projeto SWERA. In: SIMPÓSI0 BRASILEIR0 DE SENSORIAMENT0 REM0T0, 12., Goiânia, 2005. Anais. São José dos Campos: INPE, 2005.

MOM0I, A.. Projeto e Testes de Coletor Solar de Alta Eficiência. 2005. Monografia (Graduação em Engenharia Mecânica) - Escola Politécnica da Universidade de São Paulo, São Paulo.

MUNSON, B.R., YOUNG, D.F., OKIISHI, T.H.. Fundamentos da Mecânica dos Fluidos. Tradução da $4^{\mathrm{a}}$ edição americana. São Paulo: Ed. Edgard Blucher, 2002.

POTTER, M. C.; SCOTT, E. P.. Ciencias térmicas: termodinâmica, mecânica dos fluidos e transmissão de calor. São Paulo: Thomson Learning, 2006.

SILVA, J.A.C.. Potenciais aplicações de tecnologias solares em processos industriais como fonte de energia: uma revisão bibliográfica. Lorena: USP, 2014.

SONNTAG, R. E.; BORGNAKKE, C.; VAN WYLEN, G. J.. Fundamentos da Termodinâmica Clássica. Tradução da $4^{\mathrm{a}}$ Ed. São Paulo: Edgard Blucher, 1995. 